\title{
Avaliação e comparação da qualidade das águas entre o canal São Gonçalo e a Lagoa Mirim
}

Os recursos hídricos desempenham um grande papel no desenvolvimento das populações. Isso se deve ao fato de que os centros urbanos se desenvolveram historicamente, no entorno de rios e lagos. Com o aumento da degradação dos recursos hídricos, demonstrou-se uma maior necessidade de avaliar a qualidade das águas. $\mathrm{O}$ índice de qualidade das águas (IQA) foi desenvolvido pela National Sanitation Foundation (NSF), dos Estados Unidos em 1970, com o objetivo de comparar e monitorar as alterações na qualidade da água de corpos hídricos. O presente trabalho teve como objetivo avaliar e comparar a qualidade das águas entre o Canal São Gonçalo e a Lagoa Mirim por meio do monitoramento desses recursos hídricos, bem como identificar os pontos que apresentaram melhor ou pior índice de qualidade, através do cálculo do Índice de Qualidade das Águas adaptado pela Companhia Ambiental do Estado de São Paulo. O IQA demonstrou ser uma forte ferramenta de monitoramento da Lagoa Mirim e Canal São Gonçalo, visto que houve diferença considerável entre os pontos de coletas dos corpos hídricos estudados. Pode-se verificar que o ponto LM7 foi o que apresentou melhor resultado de IQA, sendo que no mês de novembro de 2017 foi de 91 , número considerado excelente pela ANA, e por consequência apresentou menor quantidade de coliformes e maior quantidade de oxigênio dissolvido. Já o ponto SG4 apresentou os piores IQA e a relação coliformes e oxigênio dissolvido foi inversamente proporcional ao apresentado no ponto LM7. Foram utilizados métodos estatísticos multivariados que são uma forte ferramenta para o monitoramento da qualidade de recursos hídricos, corroborando, para este estudo, a divisão dos pontos de monitoramento utilizados, bem como possibilitando o agrupamento destes pontos em componentes separados para melhor monitoramento de sua qualidade.

\section{Evaluation and comparison of the water quality between São Gonçalo channel and Mirim Lagoon}

\begin{abstract}
Water resources play a large role in population development. This is due to the fact that urban centres have developed, historically, around rivers and lakes. With the increase in water resources degradation, there was a greater need to assess water quality. The Water Quality Index (IQA) was developed by the National Sanitation Foundation (NSF) in the United States in 1970 to compare and monitor changes in water quality of water bodies. The objective of this study was to evaluate and compare the water quality between the Sao Gonçalo Channel and the Mirim Lagoon by means of the monitoring of these water resources, as well as to identify the points that presented better or worse quality index, by calculating the Index of Quality of Waters adapted by the Environmental Company of the State of São Paulo. The IQA proved to be a strong monitoring tool for the Mirim Lagoon and the Sao Gonçalo Channel since there was a considerable difference between the points of collection of the water bodies studied. It can be verified that the LM7 point was the one that presented the best IQA result, and in November 2017 it was 91, a number considered excellent by the National Water Agency, and consequently presented a smaller quantity of coliforms and a larger amount of Dissolved Oxygen. On the other hand, point SG4 presented the worst IQA and the ratio of coliforms and dissolved oxygen was inversely proportional to that presented in LM7. Multivariate statistical methods were used as a strong tool to monitor the quality of water resources, corroborating, for this study, the division of the monitoring points used, as well as allowing the grouping of these points into separate components for better monitoring of their quality.
\end{abstract}

Keywords: Water Resources; IQA; Quality.

Topic: Engenharia Ambiental

Reviewed anonymously in the process of blind peer.

Bruno Müller Vieira

Universidade Federal de Pelotas, Brasil

http://lattes.cnpq.br/1885554662703620

bruno.prppg@hotmail.com

Willian Cézar Nadaleti (iD)

Universidade Federal de Pelotas, Brasil

http://lattes.cnpq.br/4670559561277136

http://orcid.org/0000-0002-4727-4127

williancezarnadaletti@gmail.com

Marlon Heitor Kunst Valentini

Universidade Federal de Pelotas, Brasil

http://lattes.cnpq.br/6499660114940771

marlon.valentini@hotmail.com
Received: 10/02/2019

Approved: 25/03/2019

\section{Gabriel dos Santos}

Universidade Federal de Pelotas, Brasil

gabrielwxsantos@hotmail.com

\section{Francine Vicentini Viana}

Universidade Federal de Pelotas, Brasi

http://lattes.cnpq.br/0219375029813785

fravivi@gmail.com

Marília Guidotti Corrêa (D)

Universidade Federal de Pelotas, Brasil

http://lattes.cnpq.br/2113299633932476

http://orcid.org/0000-0001-9091-6001

mariliaguidotti@yahoo.com.br

\section{Referencing this:}

VIEIRA, B. M.; NADALETI, W. C.; VALENTINI, M. H. K.; SANTOS, G.; VIANA, F. V.; CORRÊA, M. G.. Avaliação e comparação da qualidade das águas entre o canal São Gonçalo e a Lagoa Mirim. Revista Ibero Americana de Ciências Ambientais, v.10, n.2, p.185-196, 2019. DOI: http://doi.org/10.6008/CBPC2179-6858.2019.002.0016 


\section{INTRODUÇÃO}

Os recursos hídricos desempenham um grande papel no desenvolvimento das populações. Isso se deve ao fato de que os centros urbanos se desenvolveram, historicamente, no entorno de rios e lagos. (OLIVEIRA, 2017). Consequentemente, o aumento da concentração populacional é um dos fatores que afetam a qualidade desses recursos hídricos, ou seja, através da ação antrópica os mananciais começam a apresentar problemas com a poluição de suas águas, que vão desde a contaminação por despejos domésticos até usos de insumos agrícolas, trazendo efeitos negativos ao homem e meio ambiente. Define-se como poluição das águas qualquer alteração física, química ou biológica na qualidade de mesma (SOUZA et al., 2014).

A qualidade das águas se dá através do efeito de processos que ocorrem ao longo do seu percurso, em relação a rios que possuem parte de seu leito em áreas urbanas a principal causa de degradação da qualidade das águas se dá devido ao lançamento de esgotos domésticos e industriais (OLIVEIRA et al., 2014). A Lagoa Mirim possui aproximadamente 375 mil Hectares de superfície da água, além disso, é uma das lagoas de água doce mais importante do Brasil, sendo o segundo maior corpo hídrico do país. Entretanto, sua localização geográfica abrange não somente o território brasileiro, mas também o território uruguaio, indo do extremo Sul do Brasil até o Leste de Uruguai (OLIVEIRA et al., 2015).

A ligação entre a Laguna dos Patos e a Lagoa Mirim se dá através do Canal São Gonçalo. Este caracteriza-se por ser um canal natural, que possui $70 \mathrm{~km}$ de extensão e que se localiza no estado do Rio Grande do Sul, na região sul do Brasil. Para evitar a salinização do canal e consequentemente da lagoa mirim foi construída uma barragem que impossibilita a entrada de água salgada em aproximadamente $80 \%$ do canal e em toda área da Lagoa Mirim, junto à mesma, foi construído uma eclusa que permite a passagem de embarcações (OLIVEIRA et al., 2015).

Segundo Steinke et al. (2008), a Lagoa Mirim tanto no território brasileiro quanto uruguaio vem sofrendo uma forte pressão antrópica em função das atividades agropastoris que são desenvolvidas em seu entorno sendo essa lagoa é uma valiosa fonte de economia nos dois países devido à atividade arrozeira desenvolvida na região. Além disso, a lagoa possui um ecossistema de grande importância, as áreas úmidas em seu entorno. Essas zonas úmidas são benéficas economicamente e socialmente pelo fato de ser um dos ecossistemas de maior produtividade do planeta, ponto que se comprova pela disponibilidade de água doce.

Segundo Centeno et al. (2016), o Canal São Gonçalo também tem grande papel econômico na região, desde lazer até abastecimento e pesca, porém o mesmo também vem sofrendo com a atividade antrópica às suas margens. Em um estudo desenvolvido por Grützmacher (2008) foi avaliado a presença de agrotóxicos no Canal São Gonçalo. Assim como a Lagoa Mirim, o canal apresenta grandes lavouras de arroz irrigado ao longo das suas duas margens. Como as águas utilizadas nessas lavouras são devolvidas ao São Gonçalo sem nenhum tratamento foi constatado a presença de quatro dos cinco agrotóxicos analisados.

Além do descarte dos agrotóxicos, grande parte do esgoto urbano coletado no município de pelotas, tratado ou não, acaba chegando ao São Gonçalo mesmo que indiretamente, influenciando também na 
qualidade deste canal (SILVA, 2007). Como o esgoto no geral contém material orgânico rico em nutrientes, consequentemente a qualidade das águas vai cair e um intenso processo de eutrofização poderá ocorrer (KIELING-RUBIO et al., 2014).

Assim determina-se que é de grande importância que as áreas afetadas sejam monitoradas, podendo identificar e avaliar as alterações desse sistema aquático e assim podendo lidar e controlar as fontes de poluição da melhor maneira possível. Lembrando que para desenvolver uma boa rede de monitoramento a mesma deve ser adaptada ao objetivo do estudo e tipo de recurso hídrico. Esse monitoramento pode ser realizado através da avaliação de parâmetros físicos, químicos e biológicos, onde relacionados podem indicar o uso e o destino para um determinado recurso (CENTENO et al., 2016).

Com o aumento da degradação dos recursos hídricos, demonstra-se uma maior necessidade de avaliar a qualidade das águas. O índice de qualidade das águas (IQA) foi desenvolvido pela National Sanitation Foundation (NSF), dos Estados Unidos em 1970, com o objetivo de comparar e monitorar as alterações na qualidade da água de corpos hídricos (FERREIRA et al., 2015).

Foi definido pela NSF um conjunto de nove parâmetros considerados mais representativos para a caracterização da qualidade das águas, são eles: oxigênio dissolvido (OD), coliformes termotolerantes, pH, Demanda Bioquímica de Oxigênio (DBO), nitrato, fósforo total, temperatura da água, turbidez e sólidos totais. Através do cálculo do IQA, se definem os níveis de qualidade do corpo d'água, onde segundo a classificação da NSF pode variar de zero (pior qualidade) a 100 (melhor qualidade) (FERREIRA et al., 2015).

Atualmente no Brasil o IQA é o principal índice de qualidade usado para o monitoramento da qualidade das águas. Esse índice, para os recursos hídricos brasileiros, foi adaptado pela Companhia Ambiental do Estado de São Paulo (CETESB) tendo como determinante principal a sua utilização para abastecimento público (ANA, 2017).

Determina-se que o IQA consiste em empregar variáveis que se correlacionam com as alterações ocorridas na micro bacia, podendo ser tanto alteração antrópica quanto natural. Além disso, o IQA apresenta a vantagem de resumir as variáveis analisadas, com diferentes unidades de medidas, em um único número, assim tornando-se uma comunicação de fácil entendimento (CENTENO et al., 2016).

Com o intuito de avaliar a qualidade da água e melhor entender a influência de certos parâmetros nessa qualidade, pode-se fazer uso de métodos estatísticos tais como os métodos multivariados. Esses métodos, tais como matrizes de correlação e análise de componentes principais, permitem a identificação dos possíveis fatores ou fontes responsáveis pelas variações na qualidade da água do manancial (HAIR JR., 2009). Ou seja, os métodos estatísticos multivariados podem oferecem uma interpretação simples a partir da análise de dados complexos, que ajudaria na melhor compreensão do comportamento do corpo hídrico estudado (BARROS, 2010). Outro método estatístico comumente usados para monitorar a qualidade de corpos d'água é a análise de variância (ANOVA). Através desse método é possível verificar se há diferença significativa para os dados analisados, mostrando como se comporta o corpo hídrico em questão (BILGIN, 2015). 
Sendo assim, o presente trabalho tem por objetivo avaliar e comparar a qualidade das águas entre o Canal São Gonçalo e a Lagoa Mirim por meio do monitoramento desses recursos hídricos, bem como identificar os pontos que apresentaram melhor ou pior índice de qualidade, através do cálculo do Índice de Qualidade das Águas (IQA) adaptado pela CETESB, bem como correlacionar e agrupar os pontos de monitoramento através do uso de métodos estatísticos multivariados e analisar a variância da qualidade das águas Lagoa Mirim e do Canal São Gonçalo entre os anos estudados.

\section{METODOLOGIA}

\section{Área de estudo}

Essa pesquisa foi desenvolvida abordando dois corpos hídricos, a Lagoa Mirim e Canal São Gonçalo, ambos localizados ao sul do rio grande do sul. A Lagoa Mirim se localiza na fronteira entre o sul do Brasil e o norte do Uruguai, na Planície Costeira do Rio Grande do Sul, e se liga a Lagoa dos Patos por meio do Canal São Gonçalo (OLIVEIRA et al., 2015). O canal São Gonçalo localiza-se na cidade de Pelotas e tem uma extensão de aproximadamente $76 \mathrm{~km}$, sendo este o curso hídrico principal de uma das sub-bacias da bacia hidrográfica da Lagoa Mirim, a bacia do São Gonçalo (SOUZA, 2013).

\section{Coletas e pontos de monitoramento}

Com o intuito de analisar a qualidade da água do Canal São Gonçalo, bem como da Bacia Hidrográfica da Lagoa Mirim, realizaram-se coletas mensais ao longo dos anos de 2016 e 2017 em onze pontos de monitoramento, sendo sete na Lagoa Mirim e quatro no Canal São Gonçalo, conforme descritos na Tabela 1. Esses pontos compreendem os dois lados da lagoa, bem como toda a extensão do canal, conforme apresentados na Figuras 1. As amostras coletadas foram encaminhadas para o laboratório de águas e efluentes da Agência da Lagoa mirim (LAEM), da Universidade Federal de Pelotas, onde foram realizadas as análises referentes ao IQA.

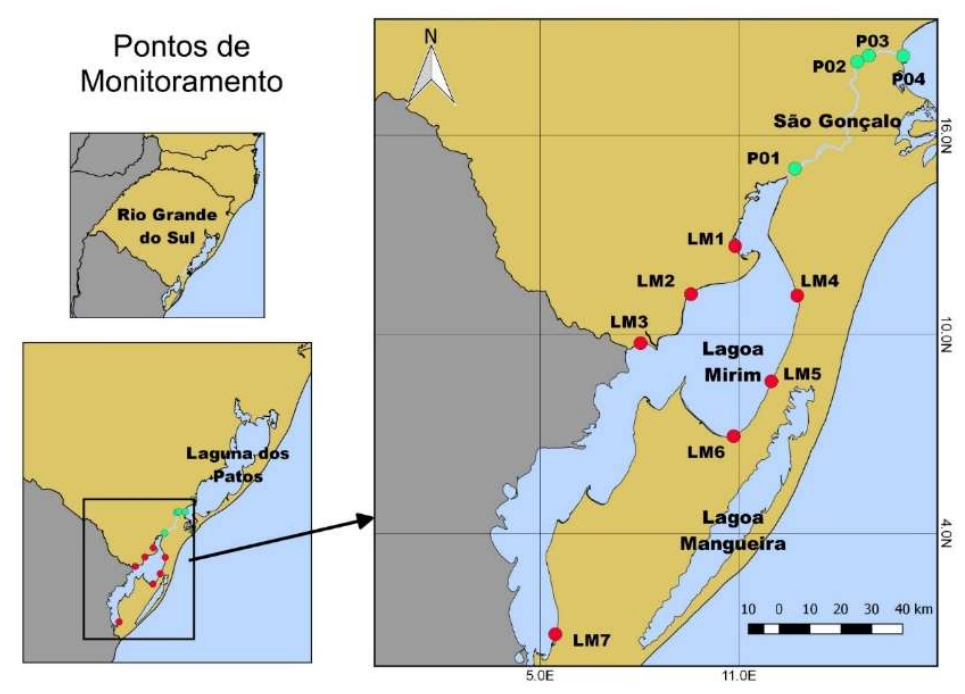

Figura 1: Localização dos Pontos de Monitoramento. 
Tabela 1: Pontos de Monitoramento.

\begin{tabular}{|l|l|l|l|}
\hline \multicolumn{2}{|l|}{ PONTOS DE MONITORAMENTO DA QUALIDADE DA ÁGUA DA LAGOA MIRIM E DO CANAL SÃO GONÇALO } \\
\hline \multicolumn{2}{|c|}{ Identificac̃o } & Coordenadas \\
\hline LM1 & Praia do Pontal & $32^{\circ} 20^{\prime} 052^{\prime \prime}$ & $52^{\circ} 49^{\prime} 21,5^{\prime \prime}$ \\
\hline LM2 & Fazenda Bretanha & $32^{\circ} 29^{\prime} 14,0^{\prime \prime}$ & $52^{\circ} 58^{\prime} 14,9^{\prime \prime}$ \\
\hline LM3 & Fazenda São Francisco & $32^{\circ} 38^{\prime} 25,6^{\prime \prime}$ & $53^{\circ} 08^{\prime} 56,8^{\prime \prime}$ \\
\hline LM4 & Capilha & $32^{\circ} 29^{\prime} 23^{\prime \prime}$ & $52^{\circ} 35^{\prime} 33^{\prime \prime}$ \\
\hline LM5 & Curral Alto & $32^{\circ} 44^{\prime} 47,41^{\prime \prime}$ & $52^{\circ} 40^{\prime} 35,99^{\prime \prime}$ \\
\hline LM6 & Vila Anselme & $32^{\circ} 54^{\prime} 31^{\prime \prime}$ & $52^{\circ} 48^{\prime} 08^{\prime \prime}$ \\
\hline LM7 & Porto de Santa Vitória & $33^{\circ} 29^{\prime} 51^{\prime \prime}$ & $53^{\circ} 26^{\prime} 09^{\prime \prime}$ \\
\hline SG1 & Santa Isabel & $31^{\circ} 76^{\prime} 14,72^{\prime \prime}$ & $52^{\circ} 28^{\prime} 82,49^{\prime \prime}$ \\
\hline SG2 & Eclusa & $31^{\circ} 78^{\prime} 25,02^{\prime \prime}$ & $52^{\circ} 31^{\prime} 89,03^{\prime \prime}$ \\
\hline SG3 & Ponte do Trem & $31^{\circ} 79^{\prime} 11,84^{\prime \prime}$ & $52^{\circ} 34^{\prime} 86,73^{\prime \prime}$ \\
\hline SG4 & Região da Barra & $31^{\circ} 82^{\prime} 03,16^{\prime \prime}$ & $52^{\circ} 39^{\prime} 27,52^{\prime \prime}$ \\
\hline
\end{tabular}

\section{Índice de qualidade da água (IQA)}

Com o objetivo de se realizar o cálculo do IQA, foram realizadas as seguintes análises: temperatura, oxigênio dissolvido, coliformes termotolerantes, pH, DBO5, nitrogênio total (NTK), fósforo total, turbidez e sólidos totais. Os resultados dessas 9 análises foram usados para o cálculo do IQA que fornece o resultado do índice de qualidade da água para cada um dos pontos de monitoramento, em cada um dos meses dos dois anos abrangidos por esse estudo. As análises feitas em laboratório seguiram a metodologia descrita em Standard methods for the examination of water and wastewater (GREENBERG, 1971).

Os nove parâmetros que compõem o IQA possuem seus respectivos pesos (w) para o cálculo desse índice. Esses pesos foram fixados em função da sua relevância para a conformação global da qualidade da água, conforme apresentados na Tabela 2. Bem como seu peso, cada parâmetro também apresenta um valor de qualidade (q), que é obtido a partir de seu gráfico de qualidade em função da sua concentração medida.

Tabela 2: Pesos relativos de cada parâmetro para o cálculo do IQA.

\begin{tabular}{|l|l|}
\hline Parâmetros do IQA e seus respectivos Pesos & Pesos \\
\hline Parâmetros & 0,17 \\
\hline Oxigênio dissolvido (OD) & 0,15 \\
\hline Coliformes termotolerantes & 0,12 \\
\hline Potencial hidrogeniônico (pH) & 0,10 \\
\hline Demanda bioquímica de oxigênio (DBO5) & 0,10 \\
\hline Temperatura & 0,10 \\
\hline Nitrogênio total & 0,10 \\
\hline Fósforo total & 0,08 \\
\hline Turbidez & 0,08 \\
\hline Sólidos Totais & \\
\hline
\end{tabular}

Fonte: ANA (2005).

Dessa forma, o IQA é calculado através do produto ponderado de seus parâmetros, conforme a Equação 1. O IQA é índice de Qualidade da Água e é dado por um número de 0 a 100, onde quanto mais próximo de 100 melhor a qualidade. 0 qi é a qualidade do i-ésimo parâmetro, também um número entre 0 e 100 que é obtido através do gráfico de qualidade em função da concentração medida. Já o wi é o peso do i-ésimo parâmetro, sendo um número entre 0 e 1 (ANA, 2005).

$$
I Q A=\prod_{i=1}^{n} q i^{w i} \quad \text { Equação } 1 .
$$


Os valores do IQA são dados em faixas e variam de acordo com os estados brasileiros. Para os fins deste artigo, relativo à área de estudo, a faixa adotada é aquela que engloba o estado do Rio Grande do Sul, conforme verificado na Tabela 3.

Tabela 3: Classificação do IQA para o estado do Rio Grande do Sul.

\begin{tabular}{|l|l|}
\hline Classificação do IQA para o RS \\
\hline Valor do IQA & Qualidade da água \\
\hline $91-100$ & Ótima \\
\hline $71-90$ & Boa \\
\hline $51-70$ & Aceitável \\
\hline $26-50$ & Ruim \\
\hline $0-25$ & Péssima \\
\hline
\end{tabular}

Fonte: ANA (2005).

\section{Análises Estatísticas}

Afim de confirmar que a quantidade de dados analisada é compatível com uma análise fatorial e análise de componentes principais (AF/ACP), primeiramente se utiliza o teste de KMO (Kaisere-MeyereOlkin). Este teste resulta em um valor de 0 a 1 conforme a adequação dos dados utilizados, sendo 0,55 o valor encontrado por este estudo. Sendo assim, descartando a hipótese de um coeficiente de correlação igual a zero, através deste teste, sendo possível dizer que a amostra de dados analisada condiz com o método pretendido (BILGIN, 2015).

Uma vez confirmada a compatibilidade dos dados com uma análise fatorial e de componentes principais, parte-se para a obtenção destes componentes. Na realização de uma análise AF/ACP são realizadas três etapas: a preparação de uma matriz de correlação, a extração dos fatores comuns e a rotação dos eixos correspondentes aos fatores comuns, afim de simplificar a interpretação da solução encontrada. (TOLEDO et al., 2002). No que concerne aos componentes principais, cada um deles é uma combinação linear de todas as variáveis originais, independentes entre si e estimadas afim de se reter o que constam nos dados iniciais. Já em relação a rotação dos eixos, essa é feita através do uso do algoritmo varimax e tem como objetivo a geração de uma matriz de componentes principais com melhor significado interpretativo, uma vez que a matriz não rotacionada nem sempre é de fácil interpretação.

Além da AF/ACP, também fez-se uma análise de variância (ANOVA) com o intuito de verificar se há variância significativa entre os dois anos estudados no que concerne ao seu índice de qualidade de água (IQA). Esse método analisa os resultados de IQA de cada um dos meses dos dois anos avaliados e mede a significância de sua variabilidade, sendo considerada como significantes aqueles resultados inferiores a um p valor de 0,05 . Para aqueles com $p$ valor maior do que 0,05 , conclui-se que não houve variação significativa entre os anos avaliados.

\section{RESULTADOS E DISCUSSÃO}

O índice de qualidade das águas foi gerado conforme descrito na metodologia, como um número entre zero e cem, sendo a melhor qualidade dada pelo extremo superior desta faixa. Os resultados obtidos no presente estudo são apresentados nas Tabelas 4 e 5. 
Tabela 4: Resultados IQA da Lagoa Mirim.

\begin{tabular}{|l|l|l|l|l|l|l|l|}
\hline \multirow{2}{*}{ Data } & IQA por pontos de coleta - Lagoa Mirim & \multicolumn{5}{l|}{ LM7 } \\
\cline { 2 - 8 } & LM1 & LM2 & LM3 & LM4 & LM5 & LM6 & LM7 \\
\hline Fev-16 & 64,00 & 75,00 & 83,00 & 71,00 & 71,00 & 76,00 & 83,00 \\
\hline Mar-16 & 69,00 & 81,00 & 77,00 & 82,00 & 90,00 & 63,00 & 81,00 \\
\hline Mai-16 & 70,00 & 63,00 & 69,00 & 70,00 & 68,00 & 70,00 & 73,00 \\
\hline Jun-16 & 68,00 & 64,00 & 61,00 & 71,00 & 60,00 & 79,00 & 83,00 \\
\hline Jul-16 & 75,00 & 78,00 & 67,00 & 59,00 & 52,00 & 61,00 & 69,00 \\
\hline Ago-16 & 89,00 & 79,00 & 89,00 & 76,00 & 60,00 & 79,00 & 81,00 \\
\hline Set-16 & 64,00 & 56,00 & 65,00 & 71,00 & 63,00 & 69,00 & 70,00 \\
\hline Out-16 & 51,00 & 56,00 & 43,00 & - & - & - & - \\
\hline Nov-16 & 60,00 & 67,00 & 73,00 & 41,00 & 37,00 & 66,00 & 72,00 \\
\hline Dez-16 & 70,00 & 56,00 & 63,00 & 68,00 & 63,00 & 53,00 & 62,00 \\
\hline Jan-17 & 61,00 & 54,00 & 52,00 & 41,00 & 44,00 & 39,00 & 43,00 \\
\hline Mar-17 & 63,00 & 60,00 & 66,00 & 72,00 & 72,00 & 53,00 & 76,00 \\
\hline Abr-17 & 58,00 & 56,00 & 62,00 & 52,00 & 52,00 & 75,00 & 78,00 \\
\hline Mai-17 & 69,00 & 57,00 & 56,00 & 62,00 & 71,00 & 46,00 & 71,00 \\
\hline Jul-17 & 63,00 & 53,00 & 61,00 & 63,00 & 53,00 & 53,00 & 65,00 \\
\hline Ago-17 & 57,00 & 54,00 & 50,00 & 51,00 & 48,00 & 49,00 & 59,00 \\
\hline Set-17 & 62,00 & 69,00 & 54,00 & 60,00 & 58,00 & 59,00 & 75,00 \\
\hline Out-17 & 61,00 & 62,00 & 58,00 & 59,00 & 58,00 & 52,00 & 63,00 \\
\hline Nov-17 & 64,00 & 72,00 & 64,00 & 67,00 & 73,00 & 71,00 & 91,00 \\
\hline Dez-17 & 63,00 & 66,00 & 78,00 & 61,00 & 73,00 & 68,00 & 79,00 \\
\hline
\end{tabular}

Conforme visualizado na Tabela 4, o melhor resultado de IQA da Lagoa Mirim foi encontrado no mês de novembro de 2017 no ponto LM7, chegando ao índice de 91, demonstrando uma ótima qualidade de água para o mês em questão. Já o pior resultado para esse mesmo corpo hídrico se deu no mês de novembro de 2016 no ponto LM5 que apresentou um resultado de IQA igual a 37, sendo classificado como ruim segundo a escala adotada para o estado do Rio Grande do Sul conforme Tabela 3.

Tabela 5: Resultados IQA do canal São Gonçalo.

\begin{tabular}{|c|c|c|c|c|}
\hline \multirow[t]{2}{*}{ Data } & \multicolumn{4}{|c|}{ IQA por pontos de coleta - São Gonçalo } \\
\hline & SG1 & SG2 & SG3 & SG4 \\
\hline Fev-16 & 74,00 & 70,00 & 72,00 & 50,00 \\
\hline Mar-16 & 77,00 & 82,00 & 58,00 & 56,00 \\
\hline Abr-16 & 69,00 & 55,00 & 61,00 & 54,00 \\
\hline Mai-16 & 77,00 & 67,00 & 76,00 & 68,00 \\
\hline Jun-16 & 63,00 & 73,00 & 77,00 & 60,00 \\
\hline Jul-16 & 83,00 & 74,00 & 64,00 & 69,00 \\
\hline Ago-16 & 83,00 & 62,00 & 58,00 & 56,00 \\
\hline Set-16 & 58,00 & 63,00 & 59,00 & 54,00 \\
\hline Out-16 & 52,00 & 55,00 & 55,00 & 51,00 \\
\hline Nov-16 & 60,00 & 46,00 & 46,00 & 45,00 \\
\hline Dez-16 & 64,00 & 60,00 & 55,00 & 62,00 \\
\hline Jan-17 & 54,00 & 71,00 & 76,00 & 56,00 \\
\hline Mar-17 & 72,00 & 58,00 & 67,00 & 58,00 \\
\hline Abr-17 & 60,00 & 62,00 & 64,00 & 57,00 \\
\hline Mai-17 & 52,00 & 64,00 & 51,00 & 59,00 \\
\hline Jul-17 & 63,00 & 69,00 & 70,00 & 61,00 \\
\hline Ago-17 & 50,00 & 49,00 & 55,00 & 44,00 \\
\hline Set-17 & 61,00 & 56,00 & 43,00 & 40,00 \\
\hline Out-17 & 82,00 & 69,00 & 71,00 & 58,00 \\
\hline Nov-17 & 77,00 & 71,00 & 72,00 & 56,00 \\
\hline Dez-17 & 64,00 & 68,00 & 65,00 & 51,00 \\
\hline
\end{tabular}

Já na Tabela 5, pode-se verificar que o melhor resultado de IQA para o canal São Gonçalo ocorreu nos meses de julho e agosto de 2016 no ponto de monitoramento SG1, mostrando um bom índice de qualidade da água para esses resultados. No que concerne ao pior resultado de IQA para este corpo hídrico, 
observa-se o ponto de monitoramento SG4 no mês de setembro de 2017 com um índice igual a 40, classificando a qualidade da água como ruim para o resultado em questão.

No que diz respeito às médias dos índices de qualidade da água, as médias de 2016 foram superiores das médias de 2017 em todos os pontos de monitoramento à exceção do ponto SG3, mostrando que, de forma geral, a qualidade dos dois recursos hídricos aqui estudados foi melhor no ano de 2016, em comparação ao ano de 2017. Esse fato aconteceu, possivelmente, pela ocorrência do El Niño no ano de 2016. Esse fenômeno climático se caracteriza, no Brasil, por um maior volume de precipitação na região sul, onde se localizam os corpos hídricos aqui estudados (SILVA et al., 2017). Com o aumento das chuvas ocorre uma diluição dos poluentes nos recursos hídricos, resultando, provavelmente, em melhores índices de qualidades. As médias obtidas para cada ponto de monitoramento da Lagoa Mirim e do canal São Gonçalo podem ser verificadas conforme as Tabelas 6 e 7 .

Tabela 6: Médias dos IQA's para os pontos de monitoramento da Lagoa Mirim.

\begin{tabular}{|l|l|l|l|}
\hline \multirow{2}{*}{ Pontos de Monitoramento } & \multicolumn{3}{l|}{ Médias IQA's - Lagoa Mirim } \\
\cline { 2 - 4 } & 2016 & 2017 & geral \\
\hline LM1 & 68,00 & 62,10 & 65,05 \\
\hline LM2 & 67,50 & 60,30 & 63,90 \\
\hline LM3 & 69,00 & 60,10 & 64,55 \\
\hline LM4 & 67,67 & 58,80 & 63,00 \\
\hline LM5 & 62,67 & 60,20 & 61,37 \\
\hline LM6 & 68,44 & 56,50 & 62,16 \\
\hline LM7 & 74,89 & 70,00 & 72,32 \\
\hline
\end{tabular}

Tabela 7: Médias IQA's para os pontos de monitoramento do canal São Gonçalo.

\begin{tabular}{|l|l|l|l|}
\hline \multirow{2}{*}{ Pontos de Monitoramentos } & \multicolumn{2}{l|}{ Médias IQA's - canal São Gonçalo } & geral \\
\cline { 2 - 4 } & 2016 & 2017 & 66,43 \\
\hline SG1 & 69,09 & 63,50 & 64,00 \\
\hline SG2 & 64,27 & 63,70 & 62,62 \\
\hline SG3 & 61,91 & 63,40 & 55,48 \\
\hline SG4 & 56,82 & 54,00 & \\
\hline
\end{tabular}

Conforme mencionado anteriormente, o IQA mede o índice de qualidade da água em relação a nove parâmetros que são analisados em laboratório e resultam em concentrações relativas à sua presença no corpo hídrico. Um IQA maior ou menor ocorre pela concentração de cada um desses parâmetros no corpo hídrico estudado, levando em consideração o peso que cada um desses parâmetros possui dentro do cálculo do IQA. Parâmetros como o oxigênio dissolvido e os coliformes termotolerantes, por possuírem um peso maior, influenciam em maior proporção do que, por exemplo, a turbidez e os sólidos totais para o resultado final do IQA. No que diz respeito a esse estudo, pode-se constatar que os piores resultados de IQA para cada um dos pontos de monitoramento estão associados, em sua maioria, aos maiores resultados de concentração de coliformes termotolerantes, bem como as menores concentrações de oxigênio dissolvido.

É possível observar que, no que concerne às suas médias, o ponto de monitoramento da Lagoa Mirim com melhor resultado de IQA foi o ponto LM7, tanto para o ano de 2016 e 2017 quanto para a média geral, bem como o ponto com o pior resultado foi o ponto LM5, no que diz respeito a sua média geral. Já para o canal São Gonçalo, o ponto SG1 obteve os melhores resultados quanto a sua média geral e sua média para o 
ano de 2016 e o ponto SG4 obteve os piores resultados de IQA tanto para as médias de 2016 e 2017, bem como para a sua média geral.

O ponto de monitoramento SG4 foi o que obteve os piores resultados, médios, de IQA em relação aos dois corpos hídricos estudados o que é corroborado pelos maiores resultados de concentração de coliformes termotolerantes, no que diz respeito às médias de 2016, 2017 e a média geral, bem como a menor média geral de oxigênio dissolvido. Já o ponto LM7 foi o que mostrou as melhores médias de IQA levando em consideração tanto a Lagoa Mirim quanto o Canal São Gonçalo, sendo esse ponto o que apresentou as menores concentrações médias de coliformes. O ponto LM7 foi o único ponto que obteve uma média geral dentro da faixa classificada como boa, bem como é o único ponto a atingir um resultado de IQA, ocorrido no mês novembro de 2017, classificado como excelente, ao considerar os resultados por mês de monitoramento.

De posse dos resultados obtidos através do cálculo do IQA, bem como do uso do software Excel ActionSat, obteve-se os resultados referentes as análises estatísticas pretendidas por este estudo, sendo estes apresentados nas tabelas 8,9 e 10, sendo estas relativas a matriz de correlação, a matriz de componentes principais rotacionada pelo algoritmo Varimax e ao teste de variância ANOVA, respectivamente.

Tabela 8: Matriz de Correlação.

\begin{tabular}{|c|c|c|c|c|c|c|c|c|c|c|c|c|}
\hline & & LM1 & LM2 & LM3 & LM4 & LM5 & LM6 & LM7 & SG1 & SG2 & SG3 & SG4 \\
\hline \multirow[t]{11}{*}{ Correlação } & LM1 & 1,000 & & & & & & & & & & \\
\hline & LM2 & ,550 & 1,000 & & & & & & & & & \\
\hline & LM3 &, 564 & ,696 & 1,000 & & & & & & & & \\
\hline & LM4 & ,550 & ,437 & ,499 & 1,000 & & & & & & & \\
\hline & LM5 & ,246 & ,392 & ,388 & ,818 & 1,000 & & & & & & \\
\hline & LM6 & ,331 & ,526 & ,666 & ,429 & ,218 & 1,000 & & & & & \\
\hline & LM7 & ,261 & ,612 & ,579 & ,591 & ,570 & ,798 & 1,000 & & & & \\
\hline & SG1 &, 552 & ,722 &, 585 &, 532 &, 383 &, 402 & ,436 & 1,000 & & & \\
\hline & SG2 & ,275 & ,374 & ,204 & ,465 & ,538 & 144 & ,194 & ,476 & 1,000 & & \\
\hline & SG3 &,- 026 &,- 073 & ,037 & ,159 & 137 & ,156 & ,013 & ,336 & ,614 & 1,000 & \\
\hline & SG4 & ,448 &,- 021 & ,058 & 320 & 191 & ,007 &,- 050 & ,441 & ,561 & ,551 & 1,000 \\
\hline
\end{tabular}

Tabela 9: Matriz de Componentes Principais.

\begin{tabular}{|l|l|l|l|}
\hline & 1 & 2 & 3 \\
\hline LM1 &, 832 &,- 007 &, 251 \\
\hline LM2 &, 776 &, 399 &,- 034 \\
\hline LM3 &, 771 &, 403 &,- 046 \\
\hline LM4 &, 362 &, 704 &, 345 \\
\hline LM5 &, 075 &, 836 &, 321 \\
\hline LM6 &, 552 &, 533 &,- 121 \\
\hline LM7 &, 408 &, 811 &,- 135 \\
\hline SG1 &, 720 &, 239 &, 426 \\
\hline SG2 &, 356 &, 793 \\
\hline SG3 &, 132 &, 100 &, 788 \\
\hline SG4 &,- 085 &,- 142 &, 859 \\
\hline
\end{tabular}

Conforme pode ser observado na Tabela 8, todos os pontos da Lagoa Mirim mais o primeiro ponto do canal São Gonçalo (SG1) possuem forte correlação entre si direta ou indiretamente, considerando como correlação indireta a correlação cruzada entre os pontos. Já para o canal São Gonçalo, podemos ver que há uma forte correlação direta entre os pontos dois, três e quatro. Para fins deste estudo, considera-se como 
fortes aquelas correlações superiores, em módulo, a 0,5. Essas correlações podem ser corroboradas, à exceção de uma possível anomalia na correlação entre os pontos LM5 e SG2, pelas localizações destes pontos (conforme mostrados na Figura1) bem como pelo tipo de atividade desenvolvida nas imediações dos mesmos, sendo que os pontos da Lagoa Mirim bem como o ponto SG1 encontram-se em área agrícola quase em sua totalidade e os pontos SG2, SG3 e SG4 encontram-se em área urbana.

No que diz respeito a extração dos componentes principais, podemos observar na Tabela 9, que foi possível extrair 3 componentes que melhor representam a variância total explicada por este tipo de análise, doravante referidos como CP1, CP2 e CP3. Conforme representado na tabela, o CP1 engloba os pontos LM1, LM2, LM3 e SG1, o CP2 engloba os pontos LM4, LM5, LM6 e LM7 e, por último, o CP3 compreende o restante dos pontos do canal São Gonçalo. A separação dos pontos de monitoramento destes recursos hídricos nos componentes principais conforme se deu pode ser explicada pela divisão dos pontos de monitoramento mostrada na Figura1 e corrobora positivamente com as suas localizações.

Tabela 10: Teste de Variância - ANOVA.

\begin{tabular}{|c|c|c|c|c|c|c|}
\hline & & Soma dos Quadrados & $\mathrm{gl}$ & Quadrado Médio & $\mathrm{F}$ & Sig. \\
\hline \multirow[t]{3}{*}{ LM1 } & Entre Grupos & 287,369 & 1 & 287,369 & 7,382 & 015 \\
\hline & Nos grupos & 661,789 & 17 & 38,929 & & \\
\hline & Total & 949,158 & 18 & & & \\
\hline \multirow[t]{3}{*}{ LM2 } & Entre Grupos & 340,450 & 1 & 340,450 & 4,931 & ,040 \\
\hline & Nos grupos & 1173,656 & 17 & 69,039 & & \\
\hline & Total & 1514,105 & 18 & & & \\
\hline \multirow[t]{3}{*}{ LM3 } & Entre Grupos & 658,316 & 1 & 658,316 & 8,467 & ,010 \\
\hline & Nos grupos & 1321,789 & 17 & 77,752 & & \\
\hline & Total & 1980,105 & 18 & & & \\
\hline \multirow[t]{3}{*}{ LM4 } & Entre Grupos & 372,400 & 1 & 372,400 & 3,518 & ,078 \\
\hline & Nos grupos & 1799,600 & 17 & 105,859 & & \\
\hline & Total & 2172,000 & 18 & & & \\
\hline \multirow[t]{3}{*}{ LM5 } & Entre Grupos & 28,821 & 1 & 28,821 & ,178 & ,679 \\
\hline & Nos grupos & 2755,600 & 17 & 162,094 & & \\
\hline & Total & 2784,421 & 18 & & & \\
\hline \multirow[t]{3}{*}{ LM6 } & Entre Grupos & 675,804 & 1 & 675,804 & 6,310 & ,022 \\
\hline & Nos grupos & 1820,722 & 17 & 107,101 & & \\
\hline & Total & 2496,526 & 18 & & & \\
\hline \multirow[t]{3}{*}{ LM7 } & Entre Grupos & 113,216 & 1 & 113,216 & ,955 & ,342 \\
\hline & Nos grupos & 2014,889 & 17 & 118,523 & & \\
\hline & Total & 2128,105 & 18 & & & \\
\hline \multirow[t]{3}{*}{ SG1 } & Entre Grupos & 266,447 & 1 & 266,447 & 2,527 & 130 \\
\hline & Nos grupos & 1792,500 & 17 & 105,441 & & \\
\hline & Total & 2058,947 & 18 & & & \\
\hline \multirow[t]{3}{*}{ SG2 } & Entre Grupos & 32,847 & 1 & 32,847 & ,417 & ,527 \\
\hline & Nos grupos & 1338,100 & 17 & 78,712 & & \\
\hline & Total & 1370,947 & 18 & & & \\
\hline \multirow[t]{3}{*}{ SG3 } & Entre Grupos & 1,834 & 1 & 1,834 & 017 & ,898 \\
\hline & Nos grupos & 1855,956 & 17 & 109,174 & & \\
\hline & Total & 1857,789 & 18 & & & \\
\hline \multirow[t]{3}{*}{ SG4 } & Entre Grupos & 67,602 & 1 & 67,602 & 1,242 & ,281 \\
\hline & Nos grupos & 925,556 & 17 & 54,444 & & \\
\hline & Total & 993,158 & 18 & & & \\
\hline
\end{tabular}

Já no que concerne ao teste de variância ANOVA, podemos ver que, apesar de as médias de 2016 serem superiores a 2017 para os resultados de IQA de todos os pontos monitorados neste estudo a exceção do ponto SG3, só há variância significativa entre os anos para os pontos LM1, LM2, LM3 e LM6 que obtiveram p valor inferior a 0,05, conforme demonstrado na coluna de significância (sig) da Tabela 10. Para o restante 
dos pontos de monitoramento, não houve variância significativa, conforme demonstrado na Tabela 10. Para o desenvolvimento deste teste de variância se usou um intervalo de confiabilidade de $95 \%$, sendo assim expresso pelo $p$ valor inferior a 0,05 para confirmação de uma variância significativa, conforme dito anteriormente. Isso se dá através da rejeição da hipótese nula de que não há significância na variabilidade dos fatores, ou seja, para p valor inferior a 0,05, para um limite de confiabilidade de 95\%, a hipótese nula é rejeitada, confirmando, assim, que a variância é significativa.

É importante citar que, em sua maioria, os maiores resultados de IQA - melhor qualidade da água estão associados a baixas concentrações de coliformes termotolerantes e altas concentrações de oxigênio dissolvido, assim como os piores IQAs decorrem do oposto no que concerne às concentrações desses parâmetros. Os outros parâmetros têm impacto menor para o resultado final, porém uma combinação deles pode afetar para mais ou para menos o índice de qualidade da água, o que faz com que essa relação entre IQA, oxigênio dissolvido e coliformes termotolerantes não seja evidenciada em todos os meses de monitoramento.

\section{CONCLUSÕES}

Pode-se concluir que a realização de análises do IQA é uma forte ferramenta de monitoramento da Lagoa Mirim e Canal São Gonçalo, visto que houve diferença considerável entre os pontos de coletas dos corpos hídricos estudados, no que diz respeito aos resultados mensais. Pode-se verificar que o ponto LM7 foi o que apresentou melhor resultado de IQA, sendo que no mês de novembro de 2017 foi de 91, número considerado excelente pela ANA, e por consequência apresentou menor quantidade de coliformes e maior quantidade de oxigênio dissolvido, induzindo que nele existe uma menor incidência de atividade antrópica no local. Já o ponto SG4 apresentou os piores IQA e a relação coliformes e oxigênio dissolvido foi inversamente proporcional ao apresentado no ponto LM7 e provavelmente ocorre maior atividade antrópica nessa região.

Conclui-se também que o uso de métodos estatísticos multivariados nos dá uma forte ferramenta para o monitoramento da qualidade de recursos hídricos, corroborando, para este estudo, a divisão dos pontos de monitoramento utilizados, bem como possibilitando o agrupamento destes pontos em componentes separados para melhor monitoramento de sua qualidade.

Dessa forma, é fundamental que o monitoramento desses pontos continue sendo estudados para que possamos no futuro compreender melhor certos fenômenos naturais que ocorrem nesses corpos hídricos e também obter um panorama geral da atividade antrópica existente nessas regiões e consequentemente alertar os órgãos de controle com a finalidade de melhorar o IQA tanto da Lagoa Mirim como o Canal São Gonçalo.

\section{REFERÊNCIAS}

ANA. Agência Nacional das Águas. Indicadores de qualidade: Índice de qualidade das águas. Brasília, 2017.
ANA. Agência Nacional das Águas. Caderno de Recursos Hídricos: 1 Panorama da qualidade das águas superficiais no Brasil. Brasília: TDA Desenho e Arte Ltda., 2005. 
BARROS, E. C. M.; ALCÂNTARA, L. O; SANTOS, M. R. P.; BUARQUE, H. L. B.; GOMES, R. B.. Análise estatística multivariada de dados de qualidade da água da Lagoa da Parangaba, Fortaleza, Ceará, Brasil. In: CONNEPI, 5. Anais. 2010.

BILGIN, A.. An assessment of water quality in the Coruh Basin (Turkey) using multivariate statistical techniques. Environmental monitoring and assessment, v.187, n.11, p.721, 2015. DOI: http://doi.org/10.1007/s10661-015-4904-9

CENTENO, L. N.; CECCONELLO, S. T.. Modificação de um índice de qualidade da água. Rev. Cient. Rural-Urcamp, Bagé, v.18, n.1, 2016.

FERREIRA, K. C. D.; LOPES, F. B.; ANDRADE, E. M.; MIIRELES, A. C. M.; SILVA, G. S.. Adaptação do índice de qualidade da água da National Sanitation foundation ao semiárido brasileiro. Revista Ciência Agronômica, v.46, n.2, p.277-286, 2015.

GRÜTZMACHER, D. D.; GRÜTZMACHER, A. D.; AGOSTINETTO, D.; LOECK, A. E.; ROMAN, R.; PEIXOTO, S. C.; ZANELLA, R. . Monitoramento de agrotóxicos em dois mananciais hídricos no sul do Brasil. Revista Brasileira de Engenharia Agrícola e Ambiental, v.12, n.6, p.632-637, 2008.

HAIR JR., J. F.; BLACK, W. C.; BABIN, B. J.; ANDERSON, R. E.; TATHAM, R. L.. Análise multivariada de dados. 6 ed. Porto Alegre: Bookman, 2009.

KIELING-RUBIO, M. A.; BENVENUTI, T.; COSTA, G. M.; PETRY, C. T.; RODRIGUES, M. A. S.; SCHMITT, J. L.; DROSTE, A.. Integrated environmental assessment of streams in the Sinos River basin in the State of Rio Grande do Sul, Brazil. Brazilian Journal of Biology, v.73, n.4, 2014. DOI: http://dx.doi.org/10.1590/1519-6984.1013

OLIVEIRA, H. A.; FERNANDES, E. H. L.; MÖLLER JUNIOR, O. O.; COLLARES, G. L.. Processos hidrológicos e hidrodinâmicos da
Lagoa Mirim. Revista Brasileira de Recursos Hídricos, v.20, n.1, p.34-45, 2015

OLIVEIRA, L. N.; SILVA, C. E.. Qualidade da água do rio poti e suas implicações para atividade de lazer em Teresina/PI. Revista Equador, v.3, n.1, p.128-147, 2014

OLIVEIRA, M. L. V. M.. Gestão de águas, territórios e desenvolvimento econômico. ACTA Geográfica, Boa Vista, v.11, n.27, p.42-61, 2017

GREENBERG, A. E.. Standard methods for the examination of water and wastewater. $23 \mathrm{ed}$. Washington: American Public Health Association (APHA), 1971.

SILVA, L. C.. Plano de gestão ambiental e social. Programa de desenvolvimento municipal integrado, 2007.

SILVA, M. P.; GALATTO, S. L.; CAMASSOLA, T. M.. A variabilidade na precipitação pluviométrica média durante o el niño 2015/2016 em Chapecó, Indaial e Urussanga, municípios do estado de Santa Catarina, Brasil. Tecnologia e Ambiente, v.23, p.108-121, 2017.

SOUZA, J. R.; MORAES, M. E. B.; SONODA, S. L.; SANTOS, H. C. R. G... A Importância da Qualidade da Água e os seus Múltiplos Usos: Caso do Rio Almada, Sul da Bahia, Brasil. Revista Eletrônica do Prodema, v.8, n.1, 2014.

SOUZA, M. F.; MEDRONHA, G. A.; MILANI, I. C. Monitoramento da qualidade da água de um manancial hídrico sob influência de atividades antrópicas, no município de Pelotas, RS/Brasil. In: SIMPÓSIO BRASILEIRO DE RECURSOS HÍDRICOS, ABRH. Anais. 2013.

STEINKE, V. A.; SAITO, C. H.. Exportação de carga poluidora para identificação de áreas úmidas sob risco ambiental na bacia hidrográfica da Lagoa Mirim. Sociedade \& Natureza, v. 20, n.2, p. $4367,2008$.

TOLEDO, L. G.; NICOLELLA, G.. Índice de qualidade de água em microbacia sob uso agrícola e urbano. Scientia Agrícola, v.59, p.181-186, 2002.

A CBPC - Companhia Brasileira de Produção Científica (CNPJ: 11.221.422/0001-03) detém os direitos materiais desta publicação. Os direitos referem-se à publicação do trabalho em qualquer parte do mundo, incluindo os direitos às renovações, expansões e disseminações da contribuição, bem como outros direitos subsidiários. Todos os trabalhos publicados eletronicamente poderão posteriormente ser publicados em coletâneas impressas sob coordenação da Sustenere Publishing, da Companhia Brasileira de Produção Científica e seus parceiros autorizados. Os (as) autores (as) preservam os direitos autorais, mas não têm permissão para a publicação da contribuição em outro meio, impresso ou digital, em português ou em tradução. 